\title{
On the curves that may be approached by trajectories of a smooth control affine system*
}

\author{
Jean-Baptiste Pomet \\ I.N.R.I.A. \\ 2004 rte des lucioles \\ B.P. 93
}

06902 SOPHIA ANTIPOLIS, France

phone: (+33) (0) 492387779

fax : $\quad(+33)(0) 492387858$

email : Jean-Baptiste.Pomet@sophia.inria.fr

July 9, 1997, revised September 3, 1998.

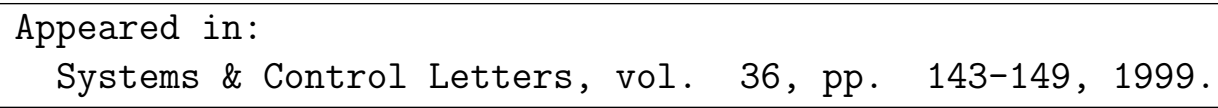

http://dx.doi.org/10.1016/S0167-6911(98)00086-3

\begin{abstract}
In this paper, we give a characterization of the set of curves that may be approached by trajectories of a smooth control-affine nonlinear system, in the topology of uniform convergence. This characterization is in terms of the drift vector field and the distribution spanned by the Lie algebra generated by the control vector fields. The characterization is valid on open sets where this distribution has constant rank. These results also characterize the support of diffusion processes with smooth coefficients.
\end{abstract}

${ }^{*}$ This work was partially supported by NSERC grant OG0036498, when the author was with Dept. of Mathematics, University of Toronto. 


\section{Introduction}

Let us consider a nonlinear control system, affine w.r.t. the controls :

$$
\dot{x}=f^{0}(x)+\sum_{k=1}^{m} u_{k} f^{k}(x),
$$

with $x \in \mathbb{R}^{n}$ (state) and $u=\left(u_{1}, \ldots, u_{m}\right) \in \mathbb{R}^{m}$ (control), and $f^{0}, f^{1}, \ldots, f^{m}$ are smooth $\left(\mathcal{C}^{\infty}\right)$ vector fields.

A parameterized curve $\gamma:[0, T] \rightarrow \mathbb{R}^{n}$ is a trajectory of the control system (1) if and only if there exist some measurable controls $t \mapsto\left(u_{1}(t), \ldots, u_{m}(t)\right)$ such that $\gamma$ is a solution of (1).

In the present paper, we aim at characterizing the curves in $\mathcal{C}^{0}\left([0, T], \mathbb{R}^{n}\right)$ that may be approached, uniformly on $[0, T]$, by trajectories of the control system (1).

It was proved by Haynes and Hermes in [2] that for driftless systems $\left(f^{0}=\right.$ $0)$, if Lie $\left\{f^{1}, \ldots, f^{m}\right\}$ has full rank, then all trajectories can be approached. An explicit construction of approximating sequences is given by Liu in [5] (see also preliminary work by Sussmann and Liu [10]), giving a method for approximate motion planning. Related results can also be found in [4] and [7]. These results can be readily extended to systems with nonzero drift if the brackets of control vector fields only still generate the whole tangent space. We investigate the case of systems with nonzero drift, and in which the rank of the distribution spanned by all the brackets between control vector fields is strictly less than $n$.

Let us mention another motivation than control theory. To the same vector fields $f^{0}, f^{1}, \ldots, f^{m}$, one may associate the diffusion process :

$$
\mathrm{d} x_{t}=f^{0}\left(x_{t}\right) \mathrm{d} t+\sum_{k=1}^{m} f^{k}\left(x_{t}\right) \circ \mathrm{d} W_{t}^{k}
$$

that is a stochastic differential equation in the sense of Stratonovich, where $\left(W^{1}, \ldots, W^{m}\right)$ is a $m$-dimensional Brownian motion. See for instance [3] for an introduction. This diffusion process induces, for all $T>0$, a measure on the set of paths $\mathcal{C}^{0}\left([0, T], \mathbb{R}^{n}\right)$. The measure of a (measurable) set of trajectories is intuitively the probability that one of them occurs as the "real" motion. The support theorem, due to Strook and Varadhan [9] states that the support of this measure is precisely the set of curves that may be approached 
by solutions of the control system (1). For this reason the present results are also of interest to diffusion processes.

The present results are valid for $\mathcal{C}^{\infty}$ vector fields away from the singular points where the rank of the distribution spanned by the Lie algebra generated by the control vector fields $f^{1}, \ldots, f^{m}$ is not locally constant. We describe exactly the curves that stay away from these points and can be approached by trajectories of our system. The somehow surprising fact is that, unlike in controllability problems, only the Lie brackets between control vector fields have an importance in this problem.

Section 2 sets the notations used in the paper. Section 3 is devoted to the main result and its proof. The reader can find in section 4 some comments on relaxing the main assumptions : smoothness and constant rank, as well as the possibility of considering other topologies than uniform convergence, in relation with motivations in control theory and in diffusion processes.

\section{Preliminaries}

For $\mathcal{F}$ an arbitrary family of smooth vector fields, and $f^{0}$ an arbitrary smooth vector field on $\mathbb{R}^{n}$, and $U$ an open subset of $\mathbb{R}^{n}$, we denote by

$$
\mathcal{T}^{0}\left(U, f^{0}, \mathcal{F}\right)
$$

the set of all continuous parameterized curves that remain in $U$ and are trajectories of a control system with $M$ controls ( $M$ a finite integer) of the form :

$$
\dot{x}=f^{0}(x)+\sum_{k=1}^{M} u_{k} X^{k}(x),
$$

where the vector fields $X^{k}$ belong to $\mathcal{F}$. To precise better what is meant by "trajectory", $\mathcal{T}^{0}\left(U, f^{0}, \mathcal{F}\right)$ is the set of all $\gamma \in \mathcal{C}^{0}([0, T], U)$ such that, for some vector fields $X^{1}, \ldots, X^{M}$ in $\mathcal{F}$, some measurable real valued functions of time $v_{1}, \ldots, v_{M}$, and for all $t \in[0, T]$,

$$
\gamma(t)-\gamma(0)=\int_{0}^{t}\left(f^{0}(\gamma(s))+\sum_{k=1}^{M} u_{k}(s) X^{k}(\gamma(s))\right) \mathrm{d} s .
$$

The elements of $\mathcal{T}^{0}\left(U, f^{0}, \mathcal{F}\right)$ are in fact obviously absolutely continuous. 
In particular, the set of all continuous trajectories of the control system (1) defined on the time-interval $[0, T]$, and that remain in the open set $U$ is $\mathcal{T}^{0}\left(U, f^{0},\left\{f^{1}, \ldots, f^{m}\right\}\right)$.

The set $\mathcal{C}^{0}([0, T], U)$ of all continuous parameterized curves on $\mathbb{R}^{n}$ that stay in $U$ is endowed with the topology of uniform convergence on the compact interval $[0, T]$, given by the distance :

$$
\left.d\left(\gamma_{1}, \gamma_{2}\right)=\sup _{t \in[0, T]} \| \gamma_{1}(t)-\gamma_{2}(t)\right) \|
$$

where $\|$.$\| is the Euclidean norm in \mathbb{R}^{n}$.

For a set of parameterized curves $\mathcal{S} \subset \mathcal{C}^{0}([0, T], U)$, we denote by $\overline{\mathcal{S}}^{\mathcal{C}^{0}}$ its topological closure in this topology.

The set of curves we are interested in is

$$
\overline{\mathcal{T}^{0}\left(U, f^{0},\left\{f^{1}, \ldots, f^{m}\right\}\right)}{ }^{\mathcal{C}^{0}},
$$

i.e. the set of continuous curves that stay in the open set $U$ of $\mathbb{R}^{n}$ and are limits of continuous trajectories of the control system (1). $U$ will be an open set on which some singularities do not occur. Note that the closure is to be taken in $\mathcal{C}^{0}([0, T], U)$ and not in $\mathcal{C}^{0}\left([0, T], \mathbb{R}^{n}\right)$, i.e. the present results do not tell when a curve that has points in the boundary of $U$ may be approached by trajectories that remain in $U$.

Finally, we denote by

$$
\operatorname{Lie}\left\{f^{1}, \ldots, f^{m}\right\} \text {, }
$$

the Lie algebra of smooth vector fields generated by $f^{1}, \ldots, f^{m}$, i.e. the $\mathcal{C}^{\infty}\left(\mathbb{R}^{n}, \mathbb{R}\right)$-module generated by all the iterated Lie Brackets made with the vector fields $f^{1}, \ldots, f^{m}$. The notation

$$
\operatorname{Lie}\left\{f^{1}, \ldots, f^{m}\right\}(x)
$$

stands for the subspace spanned by the values at $x$ of the vector fields in $\operatorname{Lie}\left\{f^{1}, \ldots, f^{m}\right\}$.

\section{The result}

We shall always restrict our attention to the situation on an open subset $U$ of $\mathbb{R}^{n}$ where the dimension of $\operatorname{Lie}\left\{f^{1}, \ldots, f^{m}\right\}(x)$ is constant, equal to a certain 
integer $r(0<r \leq n)$ :

$$
\operatorname{dim}\left(\operatorname{Lie}\left\{f^{1}, \ldots, f^{m}\right\}(x)\right)=r \quad \forall x \in U .
$$

On such an open set, the distribution $x \mapsto \operatorname{Lie}\left\{f^{1}, \ldots, f^{m}\right\}(x)$ is clearly both involutive and nonsingular, so one may apply Frobenius Theorem (see for instance [8]) :

Proposition 1 (Frobenius Theorem) Let $U$ be an open set on which condition (6) holds. There exists a locally finite covering of $U$ :

$$
U=\bigcup_{i \in I} U_{i}
$$

and for each $i$ some smooth functions $h_{i, 1}, \ldots, h_{i, n-r}$ defined on $U_{i}$ such that the level surfaces of $\left(h_{i, 1}, \ldots, h_{i, n-r}\right)$ in $U_{i}$ are integral submanifolds of $\operatorname{Lie}\left\{f^{1}, \ldots, f^{m}\right\}$ :

$$
\forall i \in I, \forall x \in U_{i}, \operatorname{Lie}\left\{f^{1}, \ldots, f^{m}\right\}(x)=\left\{\mathrm{d} h_{i, 1}(x), \ldots, \mathrm{d} h_{i, n-r}(x)\right\}^{\perp} .
$$

Note that it is not in general possible, even if $U=\mathbb{R}^{n}$, to take only one open set $U_{1}=U$ : a completely integrable distribution defines locally a nice foliation, but this is seldom true globally.

We may now state our main result :

Theorem 2 Let $U$ be an open set on which the Lie algebra generated by $f^{1}, \ldots, f^{m}$ has constant rank $r$ (condition (6)), and let the open sets $U_{i}$ and the functions $h_{i, j}$ be these given by proposition 1. A continuous parameterized curve $\gamma:[0, T] \rightarrow U$ can be approached by trajectories of (1), i.e. belongs to $\overline{\mathcal{T}}^{0}\left(U, f^{0},\left\{f^{1}, \ldots, f^{m}\right\}\right)^{C^{0}}$, if and only if

$$
h_{i, j}\left(\gamma\left(t_{2}\right)\right)-h_{i, j}\left(\gamma\left(t_{1}\right)\right)=\int_{t_{1}}^{t_{2}}\left(L_{f^{0}} h_{i, j}\right)(\gamma(s)) \mathrm{d} s
$$

for all $i, j, t_{1}, t_{2}$ such that $i \in I, 1 \leq j \leq n-r, 0 \leq t_{1}<t_{2} \leq T$, and $\gamma\left(\left[t_{1}, t_{2}\right]\right) \subset U_{i}$.

This theorem exactly describes the set $\overline{\mathcal{T}^{0}\left(U, f^{0},\left\{f^{1}, \ldots, f^{m}\right\}\right)^{\mathcal{C}^{0}}}$ of continuous curves that can be approached by trajectories of (1). However, the following description of the continuously differentiable curves that belong to this set possibly gives a better understanding of the phenomenon. It is an easy consequence of Theorem 2 and Proposition 1 : 
Corrollary 3 Let $U$ be an open set on which the constant rank condition (6) holds. A curve $\gamma \in \mathcal{C}^{1}([0, T], U)$ can be approached by trajectories of (1) if and only if, for all $t \in[0, T]$,

$$
\dot{\gamma}(t)-f^{0}(\gamma(t)) \in \operatorname{Lie}\left\{f^{1}, \ldots, f^{m}\right\}(\gamma(t)) .
$$

In other words, the set $\mathcal{C}^{1}([0, T], U) \cap \overline{\mathcal{T}^{0}\left(U, f^{0},\left\{f^{1}, \ldots, f^{m}\right\}\right)}{ }^{\mathcal{C}^{0}}$ is equal to $\mathcal{C}^{1}([0, T], U) \cap \mathcal{T}^{0}\left(U, f^{0}, \operatorname{Lie}\left\{f^{1}, \ldots, f^{m}\right\}\right)$.

It was already noticed in many places that such curves may be approached. See for instance [9, remark 4.3] or [5]. Our result states that no more differentiable curves may be approached.

Proof of theorem 2. For each fixed $i, j, t_{1}$ and $t_{2}$, the map

$$
\gamma \mapsto h_{i, j}\left(\gamma\left(t_{2}\right)\right)-h_{i, j}\left(\gamma\left(t_{1}\right)\right)-\int_{t_{1}}^{t_{2}}\left(L_{f^{0}} h_{i, j}\right)(\gamma(s)) \mathrm{d} s
$$

is continuous from $\mathcal{C}^{0}([0, T], U)$ to $\mathbb{R}$. Since any $\gamma$ in $\mathcal{T}^{0}\left(U, f^{0},\left\{f^{1}, \ldots, f^{m}\right\}\right)$ is a zero of all these maps, any $\gamma$ in $\overline{\mathcal{T}^{0}\left(U, f^{0},\left\{f^{1}, \ldots, f^{m}\right\}\right)}{ }^{\mathcal{C}^{0}}$ is also a zero of these maps. This proves that any $\gamma$ in $\overline{\mathcal{T}^{0}\left(U, f^{0},\left\{f^{1}, \ldots, f^{m}\right\}\right)} \mathcal{C}^{0}$ satisfies (9).

Let us prove the converse. Consider first a curve $\gamma \in \mathcal{C}^{0}([0, T], U)$ that satisfies condition (9) for all the required $t_{1}, t_{2}, i, j$. One may extract from the covering $\left(U_{i}\right)$ of $U$, a finite covering $\left(\mathcal{O}_{1}, \ldots, \mathcal{O}_{p}\right)$ of the compact $\gamma([0, T])$, and apply Lemma 4 below, with $q=n-r$. This gives a sequence of $\mathcal{C}^{1}$ curves $\gamma_{\varepsilon}$ that converge to $\gamma$ and satisfy also the condition (9), that may be translated, using differentiability of each $\gamma_{\varepsilon}$, into (16), that is nothing but (10). The set of continuously differentiable curves that satisfy (10) is the set of curves in $\mathcal{T}^{0}\left(U, f^{0}, \operatorname{Lie}\left\{f^{1}, \ldots, f^{m}\right\}\right)$ that are continuously differentiable. Since $\operatorname{Lie}\left\{f^{1}, \ldots, f^{m}\right\}$ is the smallest family of vector fields that contains $f^{1}, \ldots, f^{m}$ and is stable by Lie brackets and linear combinations (with coefficients $\mathcal{C}^{\infty}$ functions), we simply need to prove that for any family of vector fields $\mathcal{F}$, and any two vector fields $Y^{1}$ and $Y^{2}$ that belong to $\mathcal{F}$, any $\mathcal{C}^{1}$ curve in $\mathcal{T}^{0}\left(U, f^{0}, \mathcal{F} \cup\left\{\left[Y^{1}, Y^{2}\right]\right)\right.$ can be approached by a sequence of $\mathcal{C}^{1}$ curves in $\mathcal{T}^{0}\left(U, f^{0}, \mathcal{F}\right)$. This was essentially proved in [2] for $f^{0}=0$, and also in [4] or in $[5,10]$, and relies on the fact that, for instance, the solutions of

$$
\dot{x}=f^{0}(x)+\sum_{k=1}^{M} v_{k}(t) X^{k}(x)
$$




$$
\begin{aligned}
& \quad+\frac{v_{0}(t)}{\sqrt{\varepsilon}} \cos \frac{t}{\varepsilon} Y^{1}(x)+\frac{2}{\sqrt{\varepsilon}} \sin \frac{t}{\varepsilon} Y^{2}(x) \\
& x(0)=x_{0},
\end{aligned}
$$

with $X^{1}, \ldots, X^{M}, Y^{1}, Y^{2}$ some smooth vector fields (taken in $\mathcal{F}$ to prove the above property) and $v_{0}, \ldots, v_{M}$ some measurable functions of time, converge uniformly on $[0, T]$ to the solution of

$$
\begin{aligned}
\dot{x} & =f^{0}(x)+\sum_{k=1}^{M} v_{k}(t) X^{k}(x)+v_{0}(t)\left[Y^{1}, Y^{2}\right](x) \\
x(0) & =x_{0} .
\end{aligned}
$$

The proof is elementary and uses some integrations by parts and Gronwall Lemma.

The following lemma, whose proof is lengthy, but quite elementary, asserts that curves that satisfy condition (9) of our main theorem, but are only continuous may be uniformly approached by continuously differentiable curves satisfying the same constraint.

Lemma 4 Let $\gamma \in \mathcal{C}^{0}\left([0, T], \mathbb{R}^{n}\right)$ be a parameterized curve and $f^{0}$ be a Lipschitz continuous vector field on $\mathbb{R}^{n}$. Let $\mathcal{O}_{1}, \ldots, \mathcal{O}_{p}$ be some open sets that cover the curve $\gamma$ :

$$
\gamma([0, T]) \subset \bigcup_{i=1}^{p} \mathcal{O}_{i}
$$

Let $q$ be an integer with $1 \leq q \leq n$. Let, for each $i$, some functions $h_{i, 1}, \ldots, h_{i, q}$ be defined on the open set $\mathcal{O}_{i}$, and assumed to be differentiable with locally Lipschitz derivative (i.e. $h_{i, j} \in \mathcal{C}^{1,1}\left(\mathcal{O}_{i}, \mathbb{R}\right)$, of course $\mathcal{C}^{\infty}$-smooth is sufficient). Suppose that

1. For each $i \in\{1, \ldots, p\}$, the functions $h_{i, j}$ are independent:

$$
\operatorname{Rank}\left\{\mathrm{d} h_{i, 1}(x), \ldots, \mathrm{d} h_{i, q}(x)\right\}=q \quad \forall x \in \mathcal{O}_{i} .
$$

2. For each pair $i, i^{\prime}$ such that $\mathcal{O}_{i} \cap \mathcal{O}_{i^{\prime}}$ is nonempty, the functions $h_{i, 1}, \ldots, h_{i, q}$ define the same nonsingular foliation ${ }^{1}$ on $\mathcal{O}_{i} \cap \mathcal{O}_{i^{\prime}}$ as the functions $h_{i^{\prime}, 1}, \ldots, h_{i^{\prime}, q}$.

\footnotetext{
1 i.e. the two maps $x \mapsto\left(h_{i, 1}(x), \ldots, h_{i, q}(x)\right)$ and $x \mapsto\left(h_{i^{\prime}, 1}(x), \ldots, h_{i^{\prime}, q}(x)\right)$ from $\mathcal{O}_{i} \cap \mathcal{O}_{i^{\prime}}$ to $\mathbb{R}^{n-r}$ have the same level surfaces.
} 
3. The curve $\gamma$ is such that, for $i \in\{1, \ldots, p\}, j \in\{1, \ldots, q\}, 0 \leq t_{1}<$ $t_{2} \leq T$ one has

$$
\begin{aligned}
& \gamma\left(\left[t_{1}, t_{2}\right]\right) \subset \mathcal{O}_{i} \Longrightarrow \\
& \quad h_{i, j}\left(\gamma\left(t_{2}\right)\right)-h_{i, j}\left(\gamma\left(t_{1}\right)\right)=\int_{t_{1}}^{t_{2}} L_{f^{0}} h_{i, j}(\gamma(s)) \mathrm{d} s
\end{aligned}
$$

Then there exists a family of continuously differentiable parameterized curves $\left(\gamma_{\varepsilon}\right)_{\varepsilon>0}$ such that $\gamma_{\varepsilon}$ converges uniformly on $[0, T]$ to $\gamma$ as $\varepsilon$ goes to zero, and such that each $\gamma_{\varepsilon}$ is also covered by the open sets $\mathcal{O}_{i}$ and satisfies the property (13); this may be rephrased as :

$$
\begin{aligned}
& \gamma_{\varepsilon} \in \mathcal{C}^{1}\left([0, T], \mathbb{R}^{n}\right) \\
& \gamma_{\varepsilon}([0, T]) \subset \bigcup_{i=1}^{p} \mathcal{O}_{i}, \\
& \left.\begin{array}{c}
1 \leq j \leq q \\
\gamma_{\varepsilon}(t) \in \mathcal{O}_{i}
\end{array}\right\} \Rightarrow \frac{\mathrm{d}}{\mathrm{d} t} h_{i, j}\left(\gamma_{\varepsilon}(t)\right)=L_{f^{0}} h_{i, j}\left(\gamma_{\varepsilon}(t)\right) \\
& \lim _{\varepsilon \rightarrow 0} \gamma_{\varepsilon}=\gamma \text { in the } \mathcal{C}^{0} \text { topology. }
\end{aligned}
$$

Proof : From point 1, by possibly refining the covering $\left(\mathcal{O}_{i}\right)$, one may define on each $\mathcal{O}_{i}$ a set of coordinates in which $h_{i, 1}, \ldots, h_{i, q}$ are the first coordinates, i.e. $\xi_{i}: \mathcal{O}_{i} \rightarrow \mathbb{R}^{n}$ is given by $\xi_{i}(x)=\left(\xi_{i}^{1}(x), \ldots, \xi_{i}^{n}(x)\right)$ and

$$
1 \leq j \leq q \Rightarrow \xi_{i}^{j}=h_{i, j} \text {. }
$$

From (11), there exist some time instants $0=\tau_{0}<\tau_{1}<\cdots<\tau_{N}=T$ and some indices $i_{0}, \ldots, i_{N-1}$ such that, for all $\ell \in\{0, \ldots, N-1\}$,

$$
\gamma\left(\left[\tau_{\ell}, \tau_{\ell+1}\right]\right) \subset \mathcal{O}_{i_{\ell}} .
$$

Now, let $C_{\ell}^{j}$ be the Lipschitz continuous functions from $\xi_{i_{\ell}}\left(\mathcal{O}_{i_{\ell}}\right) \subset \mathbb{R}^{n}$ to $\mathbb{R}$ given by :

$$
C_{\ell}^{j}=\left(L_{f^{0}} \xi_{i_{\ell}}^{j}\right) \circ \xi_{\ell_{j}}^{-1}
$$

(this is the $j$ th component of the vector field $f^{0}$ in the coordinates $\xi_{i_{\ell}}$ ), and consider the following ordinary differential equation in $\mathbb{R}^{q}$

$$
\begin{aligned}
\dot{x}^{1} & =C_{\ell}^{1}\left(x^{1}, \ldots, x^{q}, \zeta^{q+1}(t), \ldots, \zeta^{n}(t)\right) \\
& \vdots \\
\dot{x}^{q} & =C_{\ell}^{q}\left(x^{1}, \ldots, x^{q}, \zeta^{q+1}(t), \ldots, \zeta^{n}(t)\right),
\end{aligned}
$$


where $\zeta^{q+1}(t), \ldots, \zeta^{n}(t)$ are some time-varying parameters. In fact this ode is only defined for values of $x$ and $\zeta$ such that $\left(x^{1}, \ldots, x^{q}, \zeta^{q+1}(t), \ldots, \zeta^{n}(t)\right)$ is in $\xi_{i_{\ell}}\left(\mathcal{O}_{i_{\ell}}\right)$. From (13) and (18), the following $\mathbb{R}^{q}$-valued function of time :

$$
\left(x^{1}=\xi_{i_{\ell}}^{1} \circ \gamma, \ldots, x^{q}=\xi_{i_{\ell}}^{q} \circ \gamma\right)
$$

is $\mathcal{C}^{1}$ and is a solution of the o.d.e. (20) on $\left[\tau_{\ell}, \tau_{\ell+1}\right]$ with $\zeta^{q+1}, \ldots, \zeta^{n}$ given by $\zeta^{j}(t)=\xi_{i_{\ell}}^{j}(\gamma(t))$. Hence, from the classical theorems on continuous dependence of the solutions with respect to the initial conditions and the parameters, for all $\eta>0$, there is a positive $\alpha_{\ell}(\eta)$ such that a solution $\left(x^{1}(t), \ldots, x^{q}(t)\right)$ of $(20)$ on the time interval $\left[\tau_{\ell}, \tau_{\ell+1}\right]$ with the parameter functions $\zeta^{q+1}, \ldots, \zeta^{n}$ such that

$$
\left|\zeta^{j}(t)-\xi_{i_{\ell}}^{j}(\gamma(t))\right| \leq \alpha_{\ell}(\eta) \quad j \in\{q+1, \ldots, n\}, t \in\left[\tau_{\ell}, \tau_{\ell+1}\right]
$$

and with an initial condition $\left(x^{1}\left(\tau_{\ell}\right), \ldots, x^{q}\left(\tau_{\ell}\right)\right)$ such that

$$
\left|x^{j}\left(\tau_{\ell}\right)-\xi_{i_{\ell}}^{j}\left(\gamma\left(\tau_{\ell}\right)\right)\right| \leq \alpha_{\ell}(\eta) \quad j \in\{1, \ldots, q\},
$$

satisfies, for all $t \in\left[\tau_{\ell}, \tau_{\ell+1}\right]$,

$$
\begin{aligned}
& \left(x^{1}(t), \ldots, x^{q}(t), \zeta^{q+1}(t), \ldots, \zeta^{n}(t)\right) \in \xi_{i_{\ell}}\left(\mathcal{O}_{i_{\ell}}\right) \\
& \left|x^{j}(t)-\xi_{i_{\ell}}^{j}(\gamma(t))\right| \leq \eta, j \in\{1, \ldots, q\} .
\end{aligned}
$$

We require in addition that $\alpha_{\ell}(\eta)<\eta$.

Let $K$ be larger than 1 and be for all $\ell$ a Lipschitz constant for $\xi_{i_{\ell+1}} \circ \xi_{i_{\ell}}^{-1}$ on $\xi_{i_{\ell}}\left(\mathcal{O}_{i_{\ell}}\right)$ (the curve $\gamma([0, T])$ is compact so that each open set $\mathcal{O}_{i}$ may, if needed, be replaced by a proper open subset of it with compact closure so that there is a Lipschitz constant on the new (smaller) $\xi_{i_{\ell}}\left(\mathcal{O}_{i_{\ell}}\right)$ for the $\mathcal{C}^{1}$ map $\xi_{i_{\ell+1}} \circ \xi_{i_{\ell}}^{-1}$ while (11) and (19) remain true). We will use the fact that, for all $x \in \xi_{i_{\ell}}\left(\mathcal{O}_{i_{\ell}}\right)$,

$$
\left\|\xi_{i_{\ell+1}} \circ \xi_{i_{\ell}}^{-1}(x)-\xi_{i_{\ell+1}}\left(\gamma\left(\tau_{\ell+1}\right)\right)\right\| \leq K\left\|x-\xi_{i_{\ell}}\left(\gamma\left(\tau_{\ell+1}\right)\right)\right\| .
$$

Then define, for $i \in\{0, \ldots, N-1\}$,

$$
\bar{\alpha}_{i}(\eta)=\frac{1}{K} \alpha_{i}\left(\frac{1}{K} \alpha_{i+1}\left(\cdots\left(\frac{1}{K} \alpha_{N-1}(\eta)\right) \cdots\right)\right) .
$$

Using (21) and the above definition of $\alpha_{\ell}(\eta)$, one deduces that these numbers $\bar{\alpha}_{i}(\eta)$ are such that

$$
\bar{\alpha}_{0}(\eta)<\bar{\alpha}_{1}(\eta)<\cdots<\bar{\alpha}_{N-1}(\eta)<\eta
$$


and such that, for $\ell \in\{0, \ldots, N-1\}$, the conditions

$$
\left.\begin{array}{l}
\left(x^{1}(t), \ldots, x^{q}(t)\right) \text { solution of }(20) \text { on }\left[\tau_{\ell}, \tau_{\ell+1}\right], \\
\mid \zeta^{j}(t)-\xi_{i_{\ell}}^{j}(\gamma(t)) \leq \alpha_{\ell}(\eta), t \in\left[\tau_{\ell}, \tau_{\ell+1}\right], j \in\{q+1, \ldots, n\}, \\
\left|x^{j}\left(\tau_{\ell}\right)-\xi_{i_{\ell}}^{j}\left(\gamma\left(\tau_{\ell}\right)\right)\right| \leq \alpha_{\ell}(\eta) \quad j \in\{1, \ldots, q\}
\end{array}\right\}
$$

imply

$$
\left.\begin{array}{l}
\left|x^{j}(t)-\xi_{i_{\ell}}^{j}(\gamma(t))\right| \leq \eta \quad, t \in\left[\tau_{\ell}, \tau_{\ell+1}\right], j \in\{1, \ldots, q\}, \\
\left|\xi_{i_{\ell+1}}\left(\xi_{i_{\ell}}^{-1}\left(x\left(\tau_{\ell+1}\right)\right)\right)-\xi_{i_{\ell+1}}^{j}\left(\gamma\left(\tau_{\ell+1}\right)\right)\right| \leq \frac{1}{K} \alpha_{\ell+1}(\eta)<\eta .
\end{array}\right\}
$$

Let us now construct the curves $\gamma_{\varepsilon}$. They will be given by :

$$
\gamma_{\varepsilon}(t)=\xi_{i_{\ell}}^{-1}\left(x_{\varepsilon, \ell}(t)\right) \quad \text { for } t \in\left[\tau_{\ell}, \tau_{\ell+1}\right] \text {, }
$$

where

$$
x_{\varepsilon, \ell}=\left(x_{\varepsilon, \ell}^{1}, \ldots, x_{\varepsilon, \ell}^{n}\right) \in \mathcal{C}^{1}\left(\left[\tau_{\ell}, \tau_{\ell+1}\right], \xi_{i_{\ell}}\left(\mathcal{O}_{i_{l}}\right)\right),
$$

and these $\mathcal{C}^{1}$ maps $x_{\varepsilon, 0}, \ldots, x_{\varepsilon, N-1}$ have the following three properties :

- For $\ell \in\{1, \ldots, N\}$,

$$
\begin{aligned}
& x_{\varepsilon, \ell}\left(\tau_{\ell}\right)=\left(\xi_{i_{\ell}} \circ \xi_{i_{\ell-1}}^{-1}\right)\left(x_{\varepsilon, \ell-1}\left(\tau_{\ell}\right)\right), \\
& \dot{x}_{\varepsilon, \ell}\left(\tau_{\ell}\right)=\left(\xi_{i_{\ell}} \circ \xi_{i_{\ell-1}}^{-1}\right)^{\prime}\left(x_{\varepsilon, \ell-1}\left(\tau_{\ell}\right)\right) \cdot \dot{x}_{\varepsilon, \ell-1}\left(\tau_{\ell}\right),
\end{aligned}
$$

where $\dot{x}_{\varepsilon, \ell}\left(\tau_{\ell}\right)$ is a half-derivative on the right, and $\dot{x}_{\varepsilon, \ell-1}\left(\tau_{\ell}\right)$ on the left.

- For $\ell \in\{0, \ldots, N-1\}$,

$$
\left\|\xi_{i_{\ell+1}}\left(\xi_{i_{\ell}}^{-1}\left(x_{\varepsilon, \ell}\left(\tau_{\ell+1}\right)\right)\right)-\xi_{i_{\ell+1}}\left(\tau_{\ell+1}\right)\right\|<\bar{\alpha}_{\ell}(\varepsilon) .
$$

- For $\ell \in\{0, \ldots, N-1\}$, and for all $t \in\left[\tau_{\ell}, \tau_{\ell+1}\right]$,

$$
\left.\begin{array}{c}
\left\|x_{\varepsilon, \ell}(t)-\xi_{i_{\ell}}(\gamma(t))\right\|<\bar{\alpha}_{\ell}(\varepsilon)<\varepsilon, \\
\left(x_{\varepsilon, \ell}^{1}, \ldots, x_{\varepsilon, \ell}^{q}\right) \text { is a solution of }(20) \\
\text { with } \zeta^{q+1}=x_{\varepsilon, \ell}^{q+1}, \ldots, \zeta^{n}=x_{\varepsilon, \ell}^{n} .
\end{array}\right\}
$$


If the family of parameterized curves $\left(\gamma_{\varepsilon}\right)$ is defined by (25) and the maps $x_{\varepsilon, \ell}$ satisfy the above six relations, then $\left(\gamma_{\varepsilon}\right)$ satisfies conditions (14) to (17) in the lemma because : (14) and (15) are consequences of (26) inside the intervals and of (27)-(28) at the junctions of these intervals ; (16) for $i=i_{\ell}$ is equivalent to (31) in the coordinates $\xi_{i_{\ell}}$, and, for each instant $t$, in virtue of the assumption on the functions $h_{i, j}$ in point 2 of the lemma, (16) is true for all $i$ such that $\gamma_{\varepsilon} \in \mathcal{O}_{i}$ if and only if it is true for one such $i$; finally, (17) is a consequence of (30) because each coordinate map $\xi_{i_{\ell}}$ is uniformly continuous in a suitable neighborhood of the curve.

To finish the proof, we only need to construct, for $\varepsilon>0$, some functions $x_{\varepsilon, \ell}$ satisfying $(26)$ to (31). Let us first construct $x_{\varepsilon, 0}$ on the interval $\left[0, \tau_{1}\right]$. For $x_{\varepsilon, 0}^{j}(t), j \in\{q+1, \ldots, n\}$, take some continuously differentiable functions (for instance some polynomials, from Stone-Weierstrass theorem), such that

$$
\left|x_{\varepsilon, 0}^{j}(t)-\xi_{i_{0}}^{j}(\gamma(t))\right|<\bar{\alpha}_{0}(\varepsilon) \quad, t \in\left[0, \tau_{1}\right] .
$$

Define then $x_{\varepsilon, 0}^{j}(t), j \in\{1, \ldots, q\}$ to be the solution of $(20)$ for $\ell=0$, with initial condition $x_{\varepsilon, 0}^{j}(0)=\xi_{i+0}(\gamma(0))$ and the time-varying parameters $\zeta^{q+1}, \ldots, \zeta^{n}$ given by $\zeta^{j}=x_{\varepsilon, 0}^{j}$. This obviously implies (31) for $\ell=0$. From (23)-(24), majorations (29)-(30) are then true for $\ell=0$.

We now extend the construction by supposing the functions $x_{\varepsilon, \ell}^{j}$ have been constructed for $0 \leq \ell \leq L$, and constructing $x_{\varepsilon, L+1}^{j}$. We assume that $x_{\varepsilon, 0}, \ldots, x_{\varepsilon, L}$ satisfy (27)-(28) for $1 \leq \ell \leq L$ and (29)-(31) for $0 \leq \ell \leq L$. We have just constructed $x_{\varepsilon, 0}$ so that this is true for $L=0$. Let us now assume it is true for a certain $L \in\{0, \ldots, N-2\}$ and construct a smooth $x_{\varepsilon, L+1}$ on the time interval $\left[\tau_{L+1}, \tau_{L+2}\right]$ so that $(27)$-(28) is true for $1 \leq \ell \leq L+1$ and (29)(31) for $0 \leq \ell \leq L+1$; in fact we only have to check (27)-(28)-(29)-(30)-(31) for $\ell=L+1$.

Take for $x_{\varepsilon, L+1}^{j}(t), j \in\{q+1, \ldots, n\}$ some smooth functions (for instance some polynomials, from Stone-Weierstrass theorem) such that

$$
\begin{aligned}
& x_{\varepsilon, L+1}^{j}\left(\tau_{L+1}\right)=\left(\xi_{i_{L+1}}^{j} \circ \xi_{i_{L}}^{-1}\right)\left(x_{\varepsilon, L}\left(\tau_{L+1}\right)\right), \\
& \dot{x}_{\varepsilon, L+1}\left(\tau_{L+1}\right)=\left(\xi_{i_{L+1}}^{j} \circ \xi_{i_{L}}^{-1}\right)^{\prime}\left(x_{\varepsilon, L+1}\left(\tau_{L+1}\right)\right) \cdot \dot{x}_{\varepsilon, L}\left(\tau_{L+1}\right), \\
& \left|x_{\varepsilon, L+1}^{j}(t)-\xi_{i_{L+1}}^{j}(\gamma(t))\right|<\bar{\alpha}_{L+1}(\varepsilon) \quad, t \in\left[\tau_{L+1}, \tau_{L+2}\right] .
\end{aligned}
$$

Note that it is possible to achieve (35) with initial conditions satisfying (33)(34) thanks to $(29)$ and the fact that $\bar{\alpha}_{L}(\varepsilon)$ is strictly less than $\bar{\alpha}_{L+1}(\varepsilon)$. 
Define then $x_{\varepsilon, L+1}^{j}(t), j \in\{1, \ldots, q\}$ to be the solution on $\left[\tau_{L+1}, \tau_{L+2}\right]$ of (20) with $\ell=L+1$, initial condition at $t=\tau_{L+1}$ given by (27) (for $\ell=L+1$, components $q+1$ to $n$ ), and the time-varying parameters $\zeta^{q+1}, \ldots, \zeta^{n}$ given by $\zeta^{j}=x_{\varepsilon, L+1}^{j}$. Then (31) is true for $\ell=L+1$ by construction, (29)-(30) from properties (23)-(24) of $\bar{\alpha}_{L}(\varepsilon)$, and (27) because we have chosen the initial conditions $x_{\varepsilon, L+1}^{j}\left(\tau_{L+1}\right)$ according to it. Let us finally check relation (28) for $\ell=L+1$ : components $q+1$ to $n$ are a direct consequence of our choice (34), but for the first $q$ components, we need the assumption on functions $h_{i, j}$ made in point 2 in Lemma 4 . From this assumption (with $\left(i, i^{\prime}\right)=\left(i_{L}, i_{L+1}\right)$ ), and from (18), the $q$ first components of $\xi_{i_{L+1}} \circ \xi_{i_{L}}^{-1}$, do not depend on its last $n-q$ arguments, and hence the $q$ first lines of the matrix $\left(\xi_{i_{L+1}} \circ \xi_{i_{L}}^{-1}\right)^{\prime}\left(x_{\varepsilon, L}\left(\tau_{L+1}\right)\right)$ have $n-q$ zeros at the end, so that the first $q$ components of the right-hand side of (28) depend only on the first $q$ components of $\dot{x}_{\varepsilon, L}\left(\tau_{L+1}\right)$. These first $q$ components of $\dot{x}_{\varepsilon, L}\left(\tau_{L+1}\right)$ are given by (20) for $\ell=L+1$ (the last $n-q$ ones result of the "free" choice of $x_{\varepsilon, L}^{q+1}, \ldots, x_{\varepsilon, L}^{n}$ at the previous step), while the first $q$ components of $\dot{x}_{\varepsilon, L+1}\left(\tau_{L+1}\right)$ in the left-hand side of (28) are given by the equation (20) for $\ell=L$. Now, the o.d.e. (20) with $\zeta^{j}=x^{j}$ is nothing but the first $q$ components of $\dot{x}=f^{0}(x)$ in the coordinates $\xi_{i_{\ell}}$, and the first $q$ components of (28) are, because they do not depend on the last components of $\dot{x}_{\varepsilon, L}\left(\tau_{L+1}\right)$, simply the coordinate change formula for the vector field $f^{0}$. This ends the construction of the functions $x_{\varepsilon, \ell}$ satisfying (26) to (31) and hence the proof of the lemma.

\section{Concluding remarks}

We have given a characterization of the set of curves that may be approached uniformly by trajectories of an affine control system. It is valid when the vector fields are smooth and the distribution spanned by the Lie algebra generated by the control vector fields has constant rank.

In practice, for control purposes, the smoothness assumption is very realistic. The constant rank assumption is more stringent. The study in the case where some singularities occur is an open question of great importance, but requiring techniques quite different from the ones used here.

We have recalled in the introduction the theorem due to Strook and Varadhan [9] that states that the support of the diffusion (2) is precisely the set $\overline{\mathcal{T}^{0}\left(U, f^{0},\left\{X^{1}, \ldots, X^{p}\right\}\right)}{ }^{\mathcal{C}^{0}}$ that we have characterized. The literature 
on diffusion processes does not usually make the assumption that the vector fields are smooth. The support theorem is usually stated under the assumption that the control vector fields are of class $\mathcal{C}^{2}$ and the drift vector field is of class $\mathcal{C}^{1}$ or less. It would therefore be interesting to relax the smoothness assumption in the present results. Smoothness, or even differentiability, of the drift vector field $f^{0}$ is never used : all results may be extended without changing a word in the proofs to the case where $f^{0}$ is only locally Lipschitz. The control vector fields on the contrary must be of class $\mathcal{C}^{\infty}$ even to state the results since otherwise the iterated Lie brackets are not defined. Also, the solutions of the diffusion (2) are not only continuous, but Hölder continuous of exponent $\alpha \in\left[0, \frac{1}{2}[\right.$, and the support theorem has recently been extended to Hölder norms in [1] and [6]. For these reasons, an extension to non-smooth control vector fields and to approximation in Hölder norms is natural. This is much less ambitious than studying the situation near singularities, even if it requires less classical tools than the ones used here, and will be treated in a forthcoming publication.

\section{Acknowledgment}

Most of the technical material underlying this paper was obtained in 198990 while the author was a post-doctoral fellow at University of Toronto (Canada). It is a pleasure for the author to thank Prof. Ivan Kupka for suggesting this question, and for many extremely fruitful discussions, and also for reminding once in a while that this should be written down !

\section{References}

[1] G. Ben-Arous, M. Gradinaru, and M. Ledoux, Hölder norms and the support theorem for diffusions, Ann. Inst. Henri Poincaré, Prob. \& Statist., 30 (1994), pp. 415-436.

[2] G. W. Haynes and H. Hermes, Nonlinear controllability via Lie theory, SIAM J. on Control, 8 (1970), pp. 450-460.

[3] N. Ikeda And S. Watanabe, Stochastic Differential equations and Diffusion Processes, vol. 24 of North-Holland Mathematical Library, North-Holand, 1989. 
[4] J. KuRZWEIL AND J. JARniK, Iterated Lie brackets in limit processes in ordinary differential equations, Results in Mathematics, 14 (1988), pp. $125-137$.

[5] W. LIU, An approximation algorithm for nonholonomic systems, SIAM J. on Control and Optim., 35 (1997), pp. 1328-1365.

[6] A. Millet And M. SAnz-Solé, A simple proof of the support theorem for diffusion processes, in Séminaire de Probabilité 28, vol. 1583 of Lect. Notes in Mathematics, Springer-Verlag, 1994.

[7] A. V. SARYCHeV, Nonlinear control systems: Topological properties of trajectory space, in Analysis of Controlled Dynamical Systems, B. Bonnard, B. Bride, J.-P. Gauthier, and I. A. K. Kupka, eds., vol. 8 of Progress in Systems and Control Theory, Brikäuser, 1991, pp. 358-371. (Proceedings of a Conf. held in Lyon, France, July, 1990).

[8] M. SpIVAK, A Comprehensive Introduction to Differential Geometry, vol. 1, Publish or Perish, Houston (U.S.A.), second ed., 1979.

[9] D. W. Stroock and S. R. S. Varadhan, On the support of diffusion processes with applications to the strong maximum principle, in Proc. Sixth Berkeley Symp. on Math. Stat. and Prob., vol. III (Probability Theory), California University Press, 1972, pp. 333-359.

[10] H. J. Sussmann And W. LiU, Limits of highly oscillatory controls and approximation of general paths by admissible trajectories, in 30th IEEE Conf. on Dec. and Control, Dec. 1991. 